\title{
Side-entrainment in a jet embedded in a sidewind
}

\author{
D. López-Cámara and A. C. Raga \\ Instituto de Ciencias Nucleares, Universidad Nacional Autónoma de México, Ap. 70-543, \\ 04510 D.F., México \\ email: diego.lopez@nucleares.unam.mx
}

\begin{abstract}
In this study, we present the results from 3D simulations in which a side-streaming motion pushes the post-bow shock into direct contact with the jet beam. This is a possible mechanism for modeling well collimated molecular jets as an atomic/ionic flow which entrains molecules initially present only in the surrounding environment.
\end{abstract}

Keywords. hydrodynamics - stars: formation - ISM: jets and outflows

\section{Introduction}

The main problem when trying to incorporate molecular, environmental material (EM), into a collimated jet is that the leading head of the jet pushes away the EM. The possibility that we study here is that a side-streaming environment pushes the bow shock wing against the jet beam.

\section{Results}

The simulations were carried out with the "Yguazú-a" code (Raga et al. 2000), solving the 3D gasdynamic equations together with a continuity/rate equation for neutral $\mathrm{H}$. We integrate an equation for a normalized passive scalar with which we distinguish between the ambient and jet medium. The jet was injected at $x=0$, with a velocity parallel to the $x$-axis. An initially neutral, top-hat jet of velocity $v_{j}$, density $n_{j}$, radius $r_{j}=10^{15} \mathrm{~cm}$ and temperature $T_{j}=10^{3} \mathrm{~K}$ moves into an initially uniform, neutral environment with a density $n_{a}=200 \mathrm{~cm}^{-3}$, temperature $T_{a}=10 \mathrm{~K}$ and sidestreaming vertical velocity $v_{a}$ (set from $y=0)$. We ran various simulations with different values of $v_{j}\left(150 \mathrm{~km} \mathrm{~s}^{-1}\right.$ for models a1;b1;c1;a3;b3;c3; and $300 \mathrm{~km} \mathrm{~s}^{-1}$ all else), $n_{j}\left(1000 \mathrm{~cm}^{-3}\right.$ for a1;b1;c1;a2;b2;c2, and $5000 \mathrm{~cm}^{-3}$ all else), and $v_{a}\left(2 \mathrm{~km} \mathrm{~s}^{-1}\right.$ for a1;a2;a3, $5 \mathrm{~km} \mathrm{~s}^{-1}$ for b1; b2; b3, and $10 \mathrm{~km} \mathrm{~s}^{-1}$ for the rest).

As an example of the flows resulting from our simulations, in Figure 1 we show $x y$-cuts showing the time-evolution of the mid-plane density stratification obtained from model a1 at various times, and for two other models at the same time. It is clear that there is a side-to-side asymmetry. This asymmetry is seen as a distortion of the leading bow shock due to the presence of the sidewind, and as a penetration of environmental material to regions close to the jet beam.

Since the total entrained material is a composition of material which was entrained directly through the bow shock, and also due to the sidewind. So, in order to recognize the material which was entrained due to the sidewind we computed the of the entrained material with $>v_{j} / 2$. The spatial distribution of the such entrained material, as well as its correspondent mass rate $\left(\dot{M}_{A M}\right)$, are shown in Figure 2. The side-entrainment 


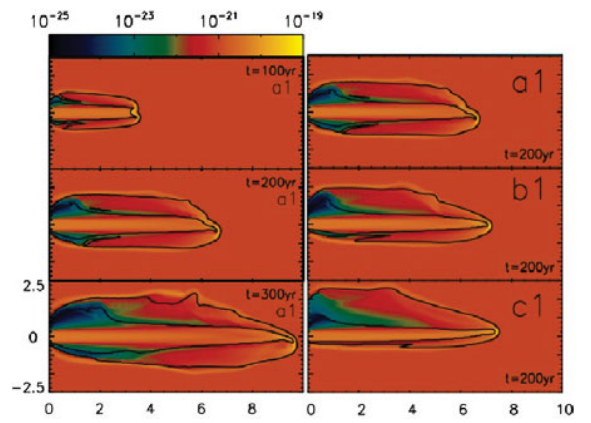

Figure 1. Density stratifications $\left(\mathrm{g} \mathrm{cm}^{-3}\right)$. The axes are labeled in units of $10^{16} \mathrm{~cm}$.

results in $\dot{M}_{A M} \sim 5 \times 10^{14} \mathrm{~g} \mathrm{~s}^{-1}$, corresponding to $\sim 0.5 \%$ of the mass loss rate of the jet. If the molecular, environmental gas is not dissociated during the process of sideentrainment, this would result in a molecular fraction of $\sim 0.5 \%$ within the jet beam, which would result in molecular column densities high enough to produce observable molecular emission.
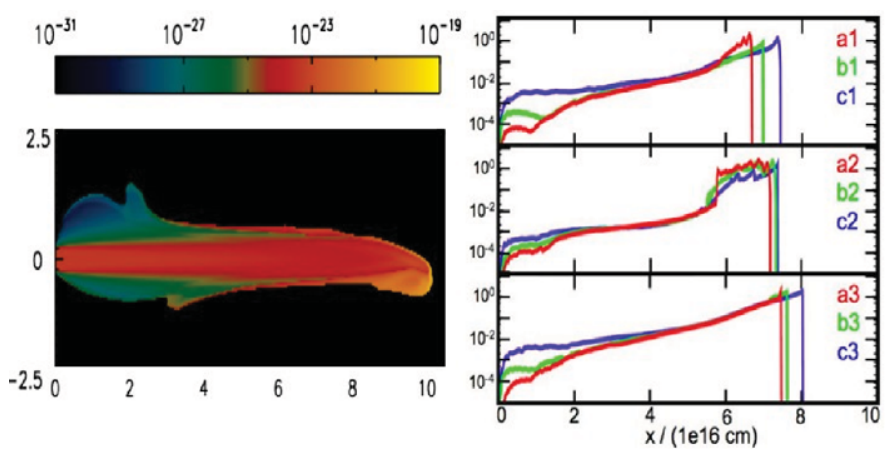

Figure 2. Left: Density stratifications $\left(\mathrm{g} \mathrm{cm}^{-3}\right)$ of the environmental mass fraction for model a1. Right: $\dot{M}_{j}$ and $\dot{M}_{A M}$ for all the models.

\section{Conclusions}

We present $3 \mathrm{D}$ numerical simulations of a jet in a sidewind. We find that for many parameter combinations the sidewind pushes the post-bow shock shell into direct contact with the jet beam. In this region of contact, side-entrainment of environmental material into the jet beam does take place. Our simulations do not include the chemistry of the entrained material, so that we are not able to see whether or not the molecules in the side-entrained material actually survive the entrainment process. However, the fact that the side-entrained material has been shocked by the slow-moving far bow shock wings, and that the region of contact between the shocked environment and the jet remains cool $\left(\sim 10^{3} \mathrm{~K}\right)$ indicates that molecules indeed might be entrained into the jet beam without being dissociated. We show that a side-streaming environment will push the post-bow shock shell into direct contact with the jet beam.

\section{References}

Raga, A. C., Navarro-González, R., \& Villagrán-Muniz, M. 2000, RMxAA, 36, 67 